\title{
UTILISATION DES SELS DE BARYUM EN VUE DU DOSAGE DES ACIDES GRAS VOLATILS DU BEURRE
}

\author{
par \\ Simone KUZDZAL-SAVOIE et W. KUZDZAL \\ Station centrale de Recherches laitières et de Technologie \\ des produits animaux, I.N.R.A., Jouy-en-Josas, Yvelines, (France)
}

\section{Introduction}

Dans une étude récente (Kuzdzal-Savoie et Kuzdzal, 1967) nous avons comparé les résultats obtenus lors du dosage des acides gras volatils du beurre; par deux méthodes différentes : la méthode de Francesco et al. (1961) et la méthode de Roos et al. (1963).

Nous proposons ci-après une nouvelle méthode, assez voisine de la méthode de Roos et al. (1963) mais adaptée au seul dosage des acides volatils.

L'étude des acides gras volatils peut présenter un intérêt dans de nombreux cas. Ainsi le schéma de répartition des acides gras volatils étant spécifiquement différent pour les laits de vache, de chèvre, de brebis, de femme, l'analyse des acides volatils peut être utilisée en vue du contrôle de la pureté de ces laits. De même, lorsqu'on se trouve en présence d'un mélange de matière grasse provenant de lait et de matière grasse naturelle d'origine différente, le dosage des acides gras volatils peut fournir d'utiles indications sur la composition du mélange, ces acides n'étant présents en quantité notable que dans la graisse du lait. Enfin, récemment, Hendrickx et Huyghebaert (1966) ont montré l'intérêt du dosage des acides volatils comme moyen de détection d'une incorporation de graisse butyrique de synthèse dans le beurre.

La méthode que nous proposons repose sur la différence de solubilité des sels de baryum des acides gras.

Cette propriété, connue depuis longtemps, est utilisée dans la détermination de l'indice de baryte. Récemment André et Henry (1964) ont décrit une technique nouvelle de détermination de cet indice. Nous nous sommes inspirés de leur travail pour préparer les savons de baryum et isoler les savons solubles, mais nous avons dosé les acides gras volatils par chromatographie en phase gazeuse. 


\section{Description de la méthode choisie}

\section{A) Préparation des savons.}

Peser à $\pm 1 \mathrm{mg}$ près, $5 \mathrm{~g}$ d'huile de beurre ou de graisse de lait dans une fiole conique de $300 \mathrm{ml}$. Incorporer un poids exactement connu d'acide valérique (environ $70 \mathrm{mg}$ ).

Ajouter ensuite $100 \mathrm{ml}$ d'une solution méthanolique d'hydroxyde de baryum 0,5 N (pour préparer cette solution, dissoudre $80 \mathrm{~g}$ d'hydroxyde de baryum $\mathrm{Ba}(\mathrm{OH})_{2}, 8 \mathrm{H}_{2} \mathrm{O}$ dans $1000 \mathrm{ml}$ de méthanol).

Chauffer sous réfrigérant ascendant au bain-marie à $80^{\circ} \mathrm{C}$ environ pendant au moins une heure. Refroidir. Homogénéiser la suspension. Ajouter $50 \mathrm{ml}$ d'eau distillée. Agiter. Placer à la glacière $\left(4^{\circ} \mathrm{C}\right)$ pendant 12 heures (une nuit). Filtrer sur papier. Ajouter quelques gouttes de phénolphtaléine en solution alcoolique dans le filtrat. Evaporer celui-ci (ou une fraction de celui-ci) à sec sur plaque chauffante à température très modérée. Réduire en poudre les savons obtenus.

B) Libération des acides volatils.

Prélever un poids $\mathrm{P}$ de savons (au minimum $40 \mathrm{mg}$ ). Les placer dans le fond d'un tube en verre de forme semblable à celui décrit par Roos et al. (1963). Ajouter un poids $5 \mathrm{P}$ de sulfate acide de sodium, un poids $\mathrm{P} / 2$ de sulfate anhydre de sodium et un volume d'hexane correspondant à $0,1 \mathrm{ml}$ pour $40 \mathrm{mg}$ de savons.

Boucher le tube au moyen d'un bouchon de caoutchouc souple dans lequel se trouve inséré un agitateur en verre atteignant le fond du tube. Triturer le mélange jusqu'à disparition de la coloration rose et exsudation de l'hexane. (Cette opération peut nécessiter quelques minutes). Incliner le tube de telle façon que la solution hexanique d'acides gras s'écoule dans la cavité ménagée sur le côté du tube. Fixer le tube dans cette position. Percer à l'aide d'une seringue de 1 à $2 \mu 1$ la membrane de caoutchouc obturant une ouverture ménagée face à la cavité et prélever la quantité de solution hexanique nécessaire pour être injectée dans l'appareil de chromatographie en phase gazeuse.

C) Analyse des acides gras volatils par chromatographie en phase gazeuse.

On a choisi les conditions opératoires suivantes :

- Appareil Aerograph, Hyfi $600 \mathrm{C}$ ou D, équipé d'un détecteur à ionisation de flamme.

- Colonne d'acier inoxydable, de $1,5 \mathrm{~m}$ de long et de $0,3 \mathrm{~cm}$ de diamètre, chargée à 15 p. 100 de DEGS (succinate de diéthylène glycol) additionné de 2 p .100 d'acide phosphorique, sur brique à feu $80 / 100$ mesh. 
- L'analyse est effectuée dans des conditions isothermiques à une température voisine de $135-140^{\circ} \mathrm{C}$. Le débit de gaz vecteur $\left(\mathrm{N}_{2}\right)$ est de $30 \mathrm{ml}$ par minute environ .

- La surface des pics est calculée au moyen de l'intégrateur Disc incorporé à l'enregistreur Honeywell utilisé.

\section{Résultats et étude critique de la méthode}

L'étude n'a porté que sur deux échantillons de beurre : le beurre $n^{\circ} 12 / 7$ fabriqué à Busseau-sur-Creuse en octobre 1966 et le beurre no 14/8 fabriqué à Arras (Somme) en novembre 1966.

Nous présentons sur la figure 1 un exemple de chromatogramme.

Dans les conditions expérimentales décrites, nous observons les pics correspondant aux acides à 4,5 (étalon interne), 6,8 et 10 atomes de carbone. Aucun autre pic n'apparaît. Très généralement le pic correspondant à l'acide à 10 atomes de carbone est très faible, et dans cette étude, nous ne considérons que les résultats se rapportant aux acides à 4,6 et 8 atomes de carbone.

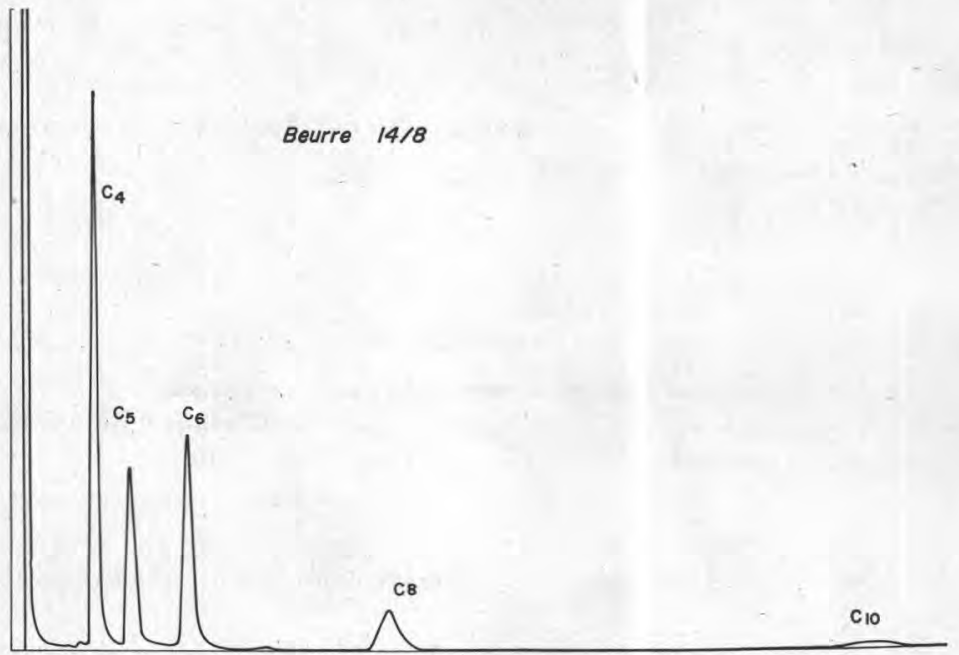

Fig. 1. - Analyse des acides gras des savons de baryum solubles. (Conditions de l'analyse : se reporter au texte).

Nous avons tenté une rapide appréciation de la valeur de la méthode à partir des données dont nous disposions. Celles-ci consistent, pour le beurre $n^{\circ} 12 / 7$ en 48 déterminations d'acide butyrique, 67 déterminations d'acide caproïque et 57 déterminations d'acide caprylique. Un nombre équivalent de données a été obtenu au moyen de chacun des appareils $\mathrm{HyFi} 600$ que nous possédions 
(C et D). Pour le beurre $n^{\circ} 14 / 8$ nous disposons d'un nombre plus faible de déterminations : 21 d'acide butyrique, 23 d'acide caproïque 17 d'acide caprylique. Toutes ces déterminations ont été obtenues avec l'appareil $\mathrm{HyFi} 600 \mathrm{C}$; seules quelques déterminations supplémentaires (5) ont été obtenues avec l'appareil $\mathrm{HyFi} 600 \mathrm{D}$.

\section{TABLEAU I}

\section{RÉSULtats anaLytiques}

(Résultats exprimés en $\mathrm{mg}$ pour $5 \mathrm{~g}$ de graisse, sans facteur de correction.)

\begin{tabular}{|c|c|c|c|c|c|}
\hline & & $\begin{array}{c}\text { Appareil } \\
\text { utilisé }\end{array}$ & $\begin{array}{c}\text { Acide } \\
\text { butyrique } \\
\mathrm{C}_{4}\end{array}$ & $\begin{array}{c}\text { Acide } \\
\text { caproïque } \\
\mathrm{C}_{6}\end{array}$ & $\begin{array}{c}\text { Acide } \\
\text { caprylique } \\
\mathrm{C}_{8}\end{array}$ \\
\hline \multirow{3}{*}{ Beurre no } & \multirow{3}{*}{$12 / 7$} & $\mathrm{C}+\mathrm{D}$ & $132,7 \pm 6,0$ & $89,3 \pm 4,8$ & $30,7 \pm 5,2$ \\
\hline & & C & $128,9 \pm 5,7$ & $92,2 \pm 4,5$ & $34,0 \pm 4,7$ \\
\hline & & D & $136,9 \pm 5,4$ & $86,6 \pm 4,2$ & $27,7 \pm 3,6$ \\
\hline \multirow{2}{*}{ Beurre $n^{\circ}$} & \multirow{2}{*}{$14 / 8$} & $\mathrm{C}$ & $138,5 \pm 6,2$ & $116,2 \pm 6,0$ & $49,9 \pm 3,8$ \\
\hline & & D & $148,0 \pm 3,0$ & $114,4 \pm 3,5$ & $46,6 \pm 3,1$ \\
\hline
\end{tabular}

\section{TABLEAU II}

Acides volatils des BeURRes anaLyses

(Résultats exprimés en $\mathrm{mg}$ p. $100 \mathrm{~g}$ de graisse : utilisation d'un facteur de correction égal à 1,1 pour l'acide butyrique.)

\begin{tabular}{c|c|c|c}
\hline \hline & $\begin{array}{c}\mathrm{C}_{4} \\
\text { Acide butyrique }\end{array}$ & $\begin{array}{c}\mathrm{C}_{6} \\
\text { Acide caproĩque }\end{array}$ & $\begin{array}{c}\mathrm{C}_{8} \\
\text { Acide caprylique }\end{array}$ \\
\cline { 2 - 3 } & $2,8-3,2$ & $1,7-1,9$ & $0,5-0,8$ \\
\hline Beurre $\mathrm{n}^{\circ} 12 / 7$ & $3,0-3,4$ & $2,2-2,4$ & $0,9-1,1$ \\
\hline \hline
\end{tabular}

$\mathrm{Au}$ cours des divers essais nous avons utilisé des quantités variables d'acide valérique s'étageant entre 50 et $120 \mathrm{mg}$ environ. Pour chaque quantité d'acide valérique ajouté, une série de 5 à 15 chromatogrammes a été obtenue. 
Dans le tableau I on a porté les résultats d'une analyse succincte des données.

On constate que la précision de la méthode est plus grande lorsqu'il s'agit de déterminer le taux d'acide caproïque ou le taux d'acide butyrique que lorsqu'il s'agit de déterminer le taux d'acide caprylique.

On constate aussi qu'il existe une légère différence selon que les déterminations sont obtenues avec l'un ou l'autre des deux appareils utilisés.

Par contre les résultats sont pratiquement indépendants de la quantité d'acide valérique ajouté (entre 50 et $120 \mathrm{mg}$ ). La figure 2 illustre ce point.
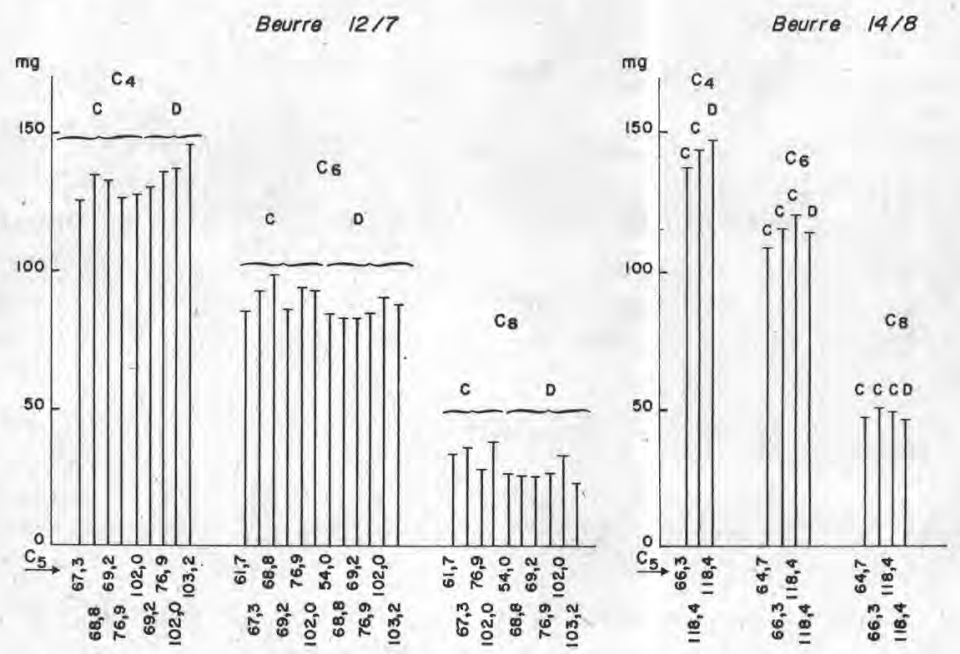

Fig. 2. - Influence de la quantité d'acide valérique ajouté, sur les résultats du dosage des acides gras volatils.

Calculées pour $100 \mathrm{~g}$ d'huile de beurre les quantités respectives des acides butyrique, caproïque et caprylique dans les deux échantillons de beurre analysés apparaissent dans le tableau II. On a introduit dans le calcul de la teneur en acide butyrique un facteur de correction égal à 1,1 . Celui-ci correspond au rapport $(0,9 / 0,8)$ des coefficients de réponse de l'appareil pour les acides butyrique et valérique relativement à l'acide pélargonique (Cf. KuzdzalSavoie et Kuzdzal, 1967).

On peut ainsi considérer que les teneurs en acide butyrique et en acide caproïque sont connues à 10 p. 100 près. La détermination de la teneur en acide caprylique comporte une plus large marge d'imprécision. Sur les chromatogrammes obtenus dans des conditions isothermiques cet acide apparaît sous la forme d'un pic nettement plus aplati que les pies correspondant aux acides à 4 , à 5 ou 
à 6 atomes de carbone, ce qui entraîne une plus grande imprécision dans la mesure de sa surface.

Nous ne pouvons affirmer que les valeurs trouvées (dans les limites de précision de la méthode) correspondent aux quantités réelles d'acide butyrique, d'acide caproïque et d'acide caprylique existant dans les beurres examinés puisque, dans l'étude présentée, les résultats obtenus ne sont pas confrontés avec des résultats obtenus par d'autres méthodes.

Toutefois les valeurs trouvées s'alignent sur les valeurs habituellement admises.

La méthode proposée a pour avantage essentiel la simplicité. (Reçu pour publication le 15 mars 1968).

\section{Résumé}

Le dosage des seuls acides gras volatils dans la graisse de lait ou dans tout mélange de graisse comportant une fraction de graisse de lait peut fournir d'utiles indications dans le domaine du contrôle.

Une méthode relativement simple, basée sur la différence de solubilité des sels de baryum des acides gras et utilisant la chromatographie en phase gazeuse directe des acides gras est proposée.

\section{Summary}

The determination of volatile fatty acids in milk fat or mixtures of milk fat and other fats may give useful informations for the milk control.

A relatively simple method, using the different solubility of the barium salts of fatty acids and the analysis of volatile fatty acids by direct gas-chromatography is proposed.

\section{RÉFÉRENCES BIBLIOGRAPHIQUES}

André E. et Henry J., 1964. Sur une étude d'analyse permettant de déterminer la quantité d'acides gras à courte chaîne contenus dans les matières grasses. C. R. hebd. Seanc. Acad. Sci., Série C., Paris, 158, 4051.

Francesco F. de, Avancini D., Maghitto C. et Gandini C., 1961. Cromatografia quantitativa in F. V. Nota III. Composizione degli acidi del grasso del latte di vacca. Riv. ital. Sostanze grasse, 41, 121.

HENDRICKX H. et HUYGHEBAERT A., 1966. Chromatografisch onderzoek van mengsels lagere vetzuren en de Reichert-Meissl-fraktie van boter en substitutievetten. Medelingen Rijksfaculteit landbauw-wetenschappen. Gent, 31, 1215.

KuzdzaL-SavoIe S. et Kuzdzal W., 1967. Le dosage des acides gras volatils du beurre par chromatographie en phase gazeuse. Le Lait, 468, 487.

Roos J. B., Versnex A, et Werdmuller G. A., 1963. Die gaschromatographische Bestimmung der niederen Fettsauren von Milchfett und deren Anwendung zum Nachwers von Fremdfetten. Kieler Milchwitrs. Forschungs, 15, 515 . 